The Impact of Large-Scale Surveys on Pulsating Star Research

ASP Conference Series, Vol. 203, 2000

L. Szabados \& D. W. Kurtż, eds.

\title{
Nonlinear Survey of RRd Models
}

\author{
R. Szabó ${ }^{1}$, Z. Kolláth ${ }^{1}$, Z. Csubry ${ }^{2}$, J. R. Buchler ${ }^{3}$
}

\begin{abstract}
We used our nonlinear hydrodynamical code with turbulent convection to model $\mathrm{RR}$ Lyrae stars. In some regime this approach produces double-mode solutions. We investigated series of models with different parameters (metallicity, luminosity, etc.). Our goal was to estimate the width of the double-mode region. The resulting tendencies can be directly compared with observational properties.
\end{abstract}

The aim of this work was to map the topology of the RRd area in the $T_{\text {eff }}-$ $L-Z-\alpha_{\nu}$ (turbulent eddy viscosity) parameter-space at a given stellar mass. An implicit, 1-D Lagrangian, nonlinear hydrodynamical code (Buchler, Kolláth, \& Marom 1997) was used that includes turbulent convection (Yecko, Kolláth, \& Buchler 1998). Sequences of models were computed with different parameters covering a reasonable range of observable and numerical parameters $(L=35-55$ $\left.L_{\odot}, Z=10^{-4}-4 \times 10^{-3}, \alpha_{\nu}=0.2-1.8\right)$. Stellar masses $\left(0.77 M_{\odot}\right)$ and hydrogen content ( $X=0.75$ ) were fixed. The following numerical (dimensionless) parameters were used: number of zones: $120, \alpha_{1}=0.41, \alpha_{c}=0.75, \alpha_{\mathrm{s}}=0.75$, $\alpha_{r m t}=1.0, \alpha_{\mathrm{d}}=4.0, \alpha_{\mathrm{p}}=\frac{2}{3}$. The calibration of the luminosity-metallicity relation is based on the empirical relation of Jurcsik (1998a) and Table 1 in Dorman's (1992) paper was used for the $[\mathrm{Fe} / \mathrm{H}]-Z$ conversion for horizontalbranch stars. We list here some apparent tendencies from our sequences:

- An increase in luminosity yields a narrower double-mode region DMR and shifts it to higher temperatures (Fig. 1). After the vanishing of doublemode pulsation a broad either-or region appears.

- Surprisingly the metallicity parameter causes only minor differences.

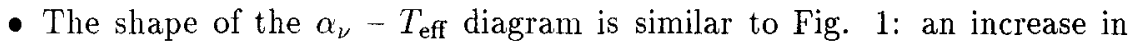
eddy viscosity pushes the DMR to higher $T_{\text {eff }}$. Another interesting feature is again the apparent either-or region at the blue edge of the DMR.

- In order to compare the location of the DMR with observational results, models of lower masses were built and similar surveys of lower masses (Bono, Castellani, \& Stellingwerf 1995; Feuchtinger 1998) were taken into account. This comparison revealed that the DMR of $0.65 M_{\odot}$ is shifted by $150-200 \mathrm{~K}$ to the blue. In this way excellent agreement can be seen with the position of observed RRd stars in M3 (Bakos \& Jurcsik 2000).

\footnotetext{
${ }^{1}$ Konkoly Observatory, P.O. Box 67 , H-1525 Budapest, Hungary

${ }^{2}$ Eötvös Loránd University, Pázmány Péter sétány, H-1117 Budapest, Hungary

${ }^{3}$ Physics Department, University of Florida, Gainesville, FL 32611, USA
} 
- Nevertheless, there is still a discrepancy between the empirical and theoretical slopes and orientations of the blue edge of the fundamental instability strip in the $L=35-55 L_{\odot}$ interval (Jurcsik, 1998b).

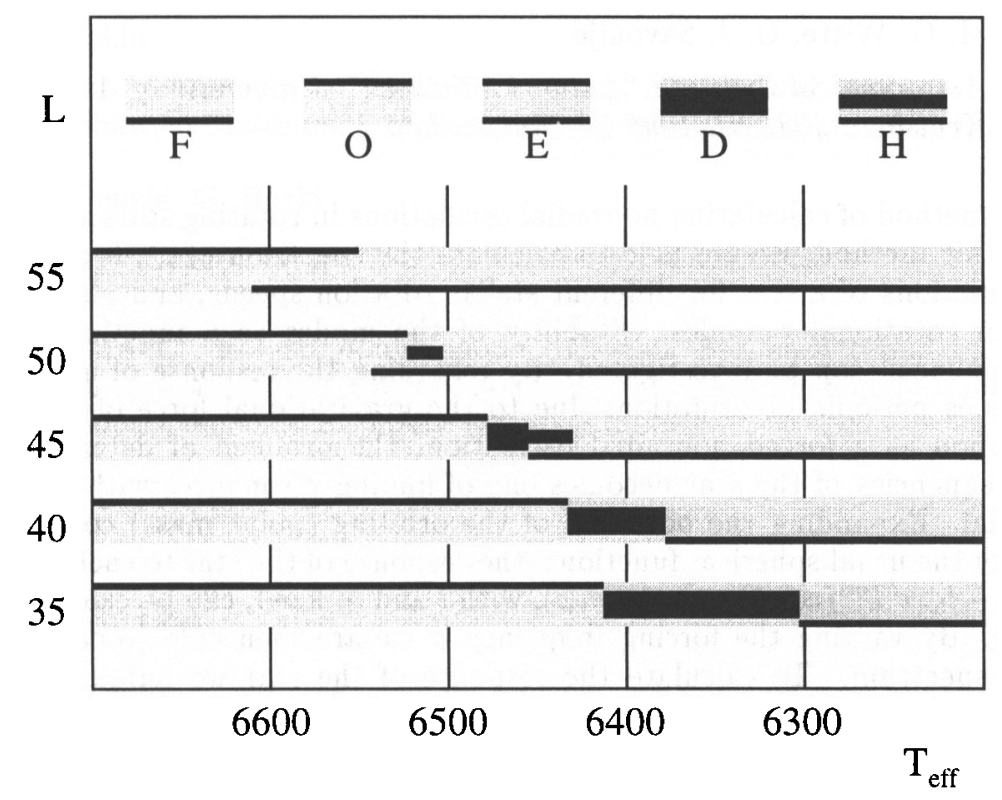

Figure 1. Double-mode (DM) instability region at $Z=0.0001$, $\alpha_{\nu}=1.0$. F: fundamental mode only, O: first overtone only, E: either fundamental mode or first overtone, D: DM only, $\mathrm{H}$ : either $\mathrm{DM}$ or fundamental mode.

Acknowledgments. This work has been supported by the Hungarian OTKA (T-026031) grant and by the National Science Foundation (AST9819608).

\section{References}

Bakos, G. Á. \& Jurcsik, J. 2000, in these proceedings, p. 255

Bono, G., Castellani, V., \& Stellingwerf, R. F. 1995, ApJ, 445, L145

Buchler, J. R., Kolláth, Z., \& Marom, A. 1997, ApJS, 253, 139

Dorman, B. 1992, ApJS, 81, 221

Feuchtingter, M. U., 1998, A\&A, 337, 29

Jurcsik, J. 1998a, A\&A, 333, 571

Jurcsik, J. 1998b, in Proc. IAU Symp. 181, Sounding Solar \& Stellar Interiors, Poster Volume, ed. J. Provost \& F.-X. Schmider (Nice: Obs. de la Coté d'Azur), 261

Yecko, P. A., Kolláth, Z., \& Buchler, J. R. 1998, A\&A, 336, 553 